\title{
The WMO SDS-WAS Regional Center for Northern Africa, Middle East and Europe
}

\author{
Sara Basart ${ }^{1, *}$, Slobodan Nickovic ${ }^{2}$,Enric Terradellas ${ }^{3}$, Emilio Cuevas $^{4}$, Carlos Pérez García-Pando ${ }^{1}$, Gerardo \\ García-Castrillo $^{3}$, Ernest Werner ${ }^{3}$ and Francesco Benincasa ${ }^{1}$ \\ ${ }^{1}$ Earth Sciences Department, Barcelona Supercomputing Center, Barcelona, Catalonia, 08034, Spain \\ 2 Republic Hydrometeorological Service of Serbia, Belgrade, Serbia \\ ${ }^{3}$ State Meteorological Agency of Spain, AEMET, Barcelona, Spain \\ ${ }^{4}$ Izaña Research Atmospheric Center-State Meteorological Agency of Spain, CIAI-AEMET, Barcelona, Spain
}

\begin{abstract}
Sand and dust storms (SDS) are an important threat to life, health, property, environment and economy in many countries, and play a significant role in different aspects of weather, climate and atmospheric chemistry. There is an increasing need for SDS accurate information and predictions to support early warning systems, and preparedness and mitigation plans. The present contribution introduces the current activities of the Regional Center for Northern Africa, Middle East and Europe of the WMO Sand and Dust Storm Warning Advisory and Assessment System (SDS-WAS). The Center has the mission is to enhance the ability of countries in the region to deliver timely and quality SDS forecasts, observations, information and knowledge to users through an international partnership of research and operational communities.
\end{abstract}

\section{INTRODUCTION}

The most important desert dust sources are located in the North Hemisphere in an area called "dust belt" (i.e. North Africa, Middle East, East Asia and North America), while other minors are in Australia, South America, and South Africa. The Saharan desert is the largest and most continuous dust source in the world. Saharan dust can be transported across the tropical North Atlantic and into the Caribbean region as well as into Europe. The Taklimakan and Gobi deserts are the major dust sources in Asia. Asian dust can be transported over the North Pacific Ocean and reach Midway and North America. For countries in and downwind of arid regions, airborne sand and dust present serious risks to the environment, property and human health. Impacts on health [1] include respiratory and cardio-vascular problems, eye infections and, in some regions, diseases such as meningitis and valley fever. Dust can carry irritating spores, bacteria, viruses and persistent organic pollutants. It can also transport nutrients to the oceans and affect marine biomass production [2]. Other impacts include negative effects on ground transport, aviation (see Fig. 1), agriculture [3] and the generation of solar energy [4]. The Intergovernmental Panel on Climate Change (IPCC) recognises dust as a major component of atmospheric aerosols, which are an "essential climate variable." Dust particles are increasingly considered by atmospheric researchers as having significant effects on weather [5] through their influence on atmospheric dynamics and chemistry [6], clouds and precipitation.
Recognising the need for international coordination of the diverse community that deals with the societal impacts of sand and dust storms, in 2007 the World Meteorological Organization took the lead and worked with international partners to develop and implement a Sand and Dust Storm Warning Advisory and Assessment System (SDS-WAS). The mission of SDS-WAS is to enhance the ability of countries to deliver timely and high- quality sand and dust storm forecasts, observations, information and knowledge to users through an international partnership of research and operational communities $[7,8]$.

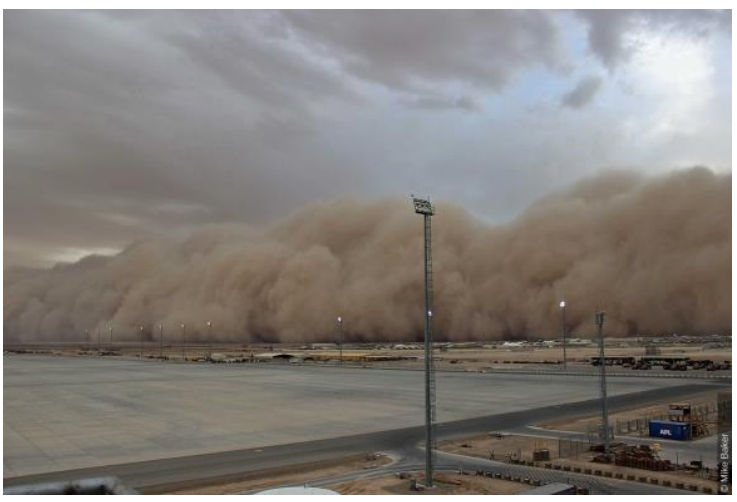

Fig. 1. Sand and dust storm Camp Bastion, near Nad-e Ali, Helmand Province, Afghanistan. (C) Mike Baker

SDS-WAS operates as an international hub of researchers, operational centres and end-users and is

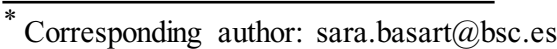


organised through regional nodes. Three nodes are currently in operation:

- Regional Node for Asia, coordinated by a Regional Center in Beijing, China, hosted by the China Meteorological Administration.

- Regional Node for Northern Africa, Middle East and Europe (NAMEE), coordinated by a Regional Center in Barcelona, Spain, hosted by the State Meteorological Agency of Spain (AEMET) and the Barcelona Supercomputing Center (BSC).

- Regional Node for Pan-America, coordinated by a Regional Center in Bridgetown, Barbados, hosted by the Caribbean Institute for Meteorology and Hydrology.

\subsection{From research to operations}

In May 2013, given the demand of many national meteorological services and the good results obtained by the SDS-WAS, which proves the feasibility and the need to begin developing operational services beyond the scope of R\&D, the WMO Executive Council designates the consortium formed by AEMET and BSC to create in Barcelona (Spain) the first Regional Specialized Meteorological Center with activity specialisation on Atmospheric Sand and Dust Forecast (RSMC-ASDF). The Center, which is called Barcelona Dust Forecast Center, started operations in March 2014, generates and distributes operational predictions for Northern Africa, Middle East and Europe. Recently, a second specialised centre in Beijing (China) has been approved and will start operations in 2019 .

\section{THE NAMEE REGIONAL CENTER}

The Regional Center for Northern Africa, Middle East and Europe (NAMEE) of the WMO SDS-WAS (http://sds-was.aemet.es), hosted by Spain, is managed by a consortium of AEMET and BSC. It was established in April 2010 to coordinate the SDS-WAS activities within this region. The Regional Centre soon evolved into a structure that hosted international and interdisciplinary research cooperation between numerous organisations in the region and beyond, including national meteorological services, environmental agencies, research groups and international organisations. All the research and dissemination activities organised around the Regional Center are centralised through its website. Therefore, the Center's web portal becomes a place where visitors can find the latest dust-related observations and the most up-to-date experimental dust forecasts. In this sense, the Regional Center is searching to promote the use of such available products, then, capacity building is at the core of the Centre's activities; more than 20 training events (https://sds-was.aemet.es/materials/training) on sand and dust observations and available forecast products have been organised in 15 countries in the region in the last few years.

\subsection{Dust Observations}

A comprehensive observational network is fundamental to any mineral dust forecasting and early warning system for real-time monitoring, validation and verification of numerical prediction models and eventual data assimilation schemes [9].

Various ground-based observational systems are in operation to monitor aerosol properties in the atmosphere [10]. While the WMO Global Atmospheric Watch (GAW) stations are measuring key aerosol variables, few of the approximately 300 GAW stations are providing near-real-time aerosol observations. In-situ measurements of particulate matter concentration and size distribution are standard in Europe, but very scarce, intermittent and rarely near-real-time available close to the main source regions. Indirect, qualitative, real-time information can be found in weather reports (as visibility, see Fig. 2) produced in synoptic stations and airfields. Ground-based aerosol remote sensing does not provide global coverage; however, its many spectral measurements of solar radiation are well suited to reliably and continuously derive aerosol optical properties. The most widely used network is AERONET (AErosol Robotic NETwork, [11]). It is a federation of ground-based remote sensing aerosol networks. It provides observations of spectral aerosol optical depth (AOD) and inversion products in geographically diverse aerosol regimes over 600 sites around the world. Most stations report in NRT and products are used at several centres for both routine and retrospective validation. Discrimination of aerosol types can be done from the aerosol optical depth and its spectral variations [12].


Fig. 3. 6-hourly visibility (in $\mathrm{km}$ ) information from METAR and SYNOP reports on 22nd March 2018. Cases of visibility reduction by sand or dust to less than $5 \mathrm{~km}$ reported in METAR or SYNOP bulletins. Brownish circles indicate stations where 'sand' or 'dust' has been explicitly reported. Triangles indicate stations where the present weather has been reported as 'haze', meaning that the visibility is reduced by particles of unspecified origin. 
Ground- and satellite-borne lidar and last generation of ceilometers are the only tools capable of inquiring about the vertical profiles of aerosol-related variables. On the one hand, research activities are indispensable, e.g., to improve our understanding of interactions of aerosols and clouds, and to develop advanced remote sensing techniques fundamentally based on lidars systems as the ones including in EARLINET (http://www.earlinet.org, last access: 18 September 2018) for the assessment of optical and microphysical aerosol properties. As a consequence of the complexity of the lidar systems, they are quite expensive; thus their number is limited, and many of them are operated by research institutes only occasionally or during dedicated field campaigns. Despite their differences from more advanced and more powerful lidars, low construction and operation cost of ceilometer, originally designed for cloud base height monitoring, have fostered their use for the quantitative study of aerosol properties. In this context, it is worthy to mention e-PROFILE initiative which is part of the EUMETNET Composite Observing System, EUCOS, managing the European networks of radar wind profilers (RWP) and automatic lidars and ceilometers for the monitoring of vertical profiles of wind and aerosols.

Satellite-borne sensors are fundamental to track the spatial distribution of dust concentrations. Sensors on board of satellite detect the radiances of various surfaces of the Earth through different spectral channels. Various satellite-sensed signals are combined: to identify and monitor dust storm in real time and to retrieve dust quantities, such as dust load, optical depth, vertical dust profiles or radiative forcing. The SEVIRI instrument onboard the MSG satellite allows generation of RGB products that offer a qualitative detection of dust clouds with a good spatial and time resolution as well as excellent and permanent coverage of the SDS-WAS Regional Center products are very important in dust monitoring, nowcasting and model verification. Particularly for the Middle East exists a specific RGB dust product base on Meteosat- 8 observations. Meteosat8 is EUMETSAT's contribution to the Indian Ocean Data Coverage (IODC) service. Furthermore, efforts of different teams aimed to develop a quantitative product for the estimation of the aerosol total-column optical depth are yielding promising results. On the other hand, products derived from instruments such as MODIS, SeaWifs, OMI, AVHRR or CALIOP onboard polar satellites and, therefore, with a lower time resolution and without permanent coverage, may help to estimate horizontal and vertical distribution, species differentiation, size distribution and optical properties of aerosols.

\subsection{Dust forecasts and its evaluation}

One of the most important activities of the Regional Center is the dust model intercomparison and forecast evaluation, which is deemed an indispensable service to the users and an invaluable tool to assess model skills. Currently, the Regional Center collects daily dust forecasts from models run by eleven partners (BSC, CNR-ISAC, ECMWF, EMA, FMI, NASA, NCEP, NOA, SEEVCCC, TNO and UK MetOffice). A multimodel ensemble has also been set up to provide addedvalue products to the users (Fig. 3).

The first problem to address the dust model evaluation is the scarcity of suitable routine observations near the Sahara, the world's largest source of mineral dust. The current routine evaluation of dust predictions within the Regional Center is focused on total-column dust optical depth (DOD) and uses remote-sensing retrievals from sun-photometric (AERONET) and satellite (MODIS) measurements.
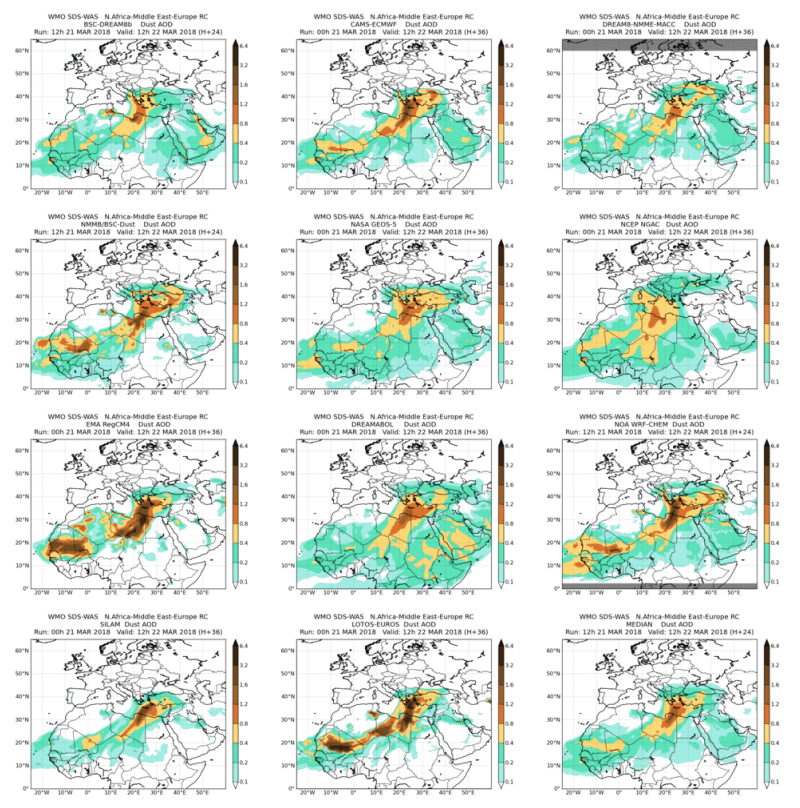

Fig. 3. Dust forecast for $22^{\text {nd }}$ March 2018 at 12 UTC. The image displays the eleven dust forecast models participating in the SDS-WAS model intercomparison. The last panel corresponds to the SDS-WAS multi-model product.

However, most users of dust forecasts are interested in the concentration near the surface (in the air we breathe) rather than in the total column content. Therefore, evaluation of the predicted surface concentration is also necessary particularly in or downwind desert dust sources in North Africa and the Middle East. Since the data sets of weather records have an excellent spatial and temporal coverage, observations of horizontal visibility included in meteorological reports (see Fig. 2) are used as an alternative way to monitor dust events in NRT and to qualitatively evaluate the dust forecasts. In this context, the initiative of the International Network to study Deposition and Atmospheric composition in Africa (INDAFF, [13]) to establish permanent measuring stations of PM10 in the Sahel is extremely important. Unfortunately, these observations are not available for operational purposes (i.e. near-real-time availability, NRT). National and regional air quality networks in North Africa and the Middle East can supply NRT PM10 observations after applying a dust-filtering [13]. 
More recently, the Regional Center started working in the evaluation of vertical profiles within the EU H2020 ACTRIS-2 project. The free troposphere contribution to AOD and the altitude of lofted layers are provided thanks to the vertical profiling capability of the lidar/ceilometer technique (see Section 2.1). Currently, a lidar located in Dakar (Senegal, managed by Lille University), and three ceilometers in Santa Cruz de Tenerife (Canary Islands, Spain; managed by AEMETCIAI), Granada (Spain; managed by University of Granada) and Montseny (Barcelona, Spain; managed by CSIC-IDAEA) provide near-real-time (NRT) vertical profiles of aerosols, which are qualitative compared with those simulated by models.

\section{OTHER RELATED INITIATIVES}

Over the past decade, there has been a growing recognition on weather, climate and ecosystems, along with their substantial adverse impact upon life, health, property, environment, economy and other strategic sectors in many countries. There is an increasing need for SDS accurate information and predictions to support early warning systems, and preparedness and mitigation plans. In alignment with the mission of the WMO Sand and Dust Storm Warning Advisory and Assessment System, recently an international consortium participates in the EU-COST Action International Network to Encourage the Use of Monitoring and Forecasting dust Products (inDust, www.cost-indust.eu). inDust has the overall objective to establish a network involving research institutions, service providers and potential end users of information on airborne dust. Otherwise, the EU Dust Storms Assessment for the development of useroriented Climate Services in Northern Africa, Middle East and Europe (DustClim) project searches to develop dust-related services tailored to specific socio-economic sectors based on an advanced dust high-resolution regional model reanalysis for Northern Africa, Middle East and Europe.

\section{Acknowledgements}

Special thanks to all researchers, data providers and collaborators of the WMO SDS-WAS NA-ME-E Regional Node. The authors would like to acknowledge the H2020 Aerosols, Clouds, and Trace gases Research InfraStructure (ACTRIS-2, grant number 654109), DustClim (which is part of ERA4CS) and the COST Action inDust (COST Action CA16202). Sara Basart and Carlos Pérez García-Pando also acknowledge the AXA Research Fund for funding aerosol research at the Barcelona Supercomputing Center through the AXA Chair on Sand and Dust Storms.

\section{References}

1. Mallone, S. et al., Saharan dust and associations between particulate matter and daily mortality in Rome, Italy. Environmental health perspectives, 119(10), 1409, (2011)
2. Jickells, T. D. et al., Global iron connections between desert dust, ocean biogeochemistry, and climate. Science, 308(5718), 67-71, (2005)

3. Stefanski, R., \& Sivakumar, M. V. K., Impacts of sand and dust storms on agriculture and potential agricultural applications of a SDS WS. In IOP Conference Series: Earth and Environmental Science, 7(1), IOP Publishing. (2009)

4. Schroedter-Homscheidt, M. et al., Aerosols for concentrating solar electricity production forecasts: requirement quantification and ECMWF/MACC aerosol forecast assessment. Bulletin of the American Meteorological Society, 94(6), 903-914, (2012)

5. Pérez, C., et al., Interactive dust-radiation modeling: A step to improve weather forecasts. Journal of Geophysical Research: Atmospheres, 111(D16) (2006)

6. Bauer, S. E., \& Koch, D., Impact of heterogeneous sulfate formation at mineral dust surfaces on aerosol loads and radiative forcing in the Goddard Institute for Space Studies general circulation model. Journal of Geophysical Research: Atmospheres, 110(D17), (2005)

7. Nickovic, S., et al., SDS-WAS Science and Implementation Plan: 2011-2015, World Meteorological Organization, Geneva (2014)

8. Terradellas, E., et al. Airborne dust: a hazard to human health, environment and society, World Meteorological Bulletin, 64(2), 42-46 (2015)

9. Benedetti, A., et al., Operational dust prediction. Mineral Dust, Springer, Dordrecht, 223-265, (2014).

10. WMO, WMO/GAW Aerosol Measurement Procedures, Guidelines and Recommendations ( $2^{\text {nd }}$ Edition, WMO Technical Report (2016)

11. Holben, B. N, et al., An emerging ground-based aerosol climatology: Aerosol optical depth from AERONET. Geophysical Research, 106(D11), 12067-12097 (2001)

12. Basart et al., Aerosol characterization in Northern Africa, Northeastern Atlantic, Mediterranean Basin and Middle East from direct-sun AERONET observations. Atmospheric Chemistry and Physics, 9(21), 8265-8282 (2009)

13. Marticorena, B., et al. Mineral dust over west and central Sahel: Seasonal patterns of dry and wet deposition fluxes from a pluriannual sampling (2006-2012). Journal of Geophysical Research: Atmospheres, 2017, 122(2), 1338-1364, (2017)

14. Pey, J., et al., African dust outbreaks over the Mediterranean Basin during 2001-2011: PM 10 concentrations, phenomenology and trends, and its relation with synoptic and mesoscale meteorology. Atmospheric Chemistry and Physics, 13(3), 13951410, (2013) 